•生物编目・

\title{
《墨尔本法规》中的“后选模式”和 “原白”概念及其应用
}

\author{
朱相云 ${ }^{*}$ \\ (中国科学院植物研究所系统与进化植物学国家重点实验室, 北京 100093)
}

\section{The two terms "lectotype" and "protologue" in Melbourne Code and their applications}

\author{
Xiangyun $\mathrm{Zhu}^{*}$ \\ State Key Laboratory of Systematic and Evolutionary Botany, Institute of Botany, Chinese Academy of Sciences, Beijing \\ 100093
}

判断一个新分类群的名称是否合格发表, 必须 基于原作者发表论文(论著)的原白(protologue)。又 由于分类群的名称依附于与其关联的命名模式 (nomenclatural type), 因此, 模式指定(type designation)在分类学研究中有着举足轻重的作用。本文作 者在阅读新近发表的植物分类学研究论文, 特别是 有关分类群修订的文章时，遇到越来越多的“后选 模式指定”(lectotypification)。详细考证之后发现诸 多错误, 主要体现在对后选模式及原白概念的错误 理解上。在《国际藻类、菌物和植物命名法规》 (International Code of Nomenclature for Algae, Fungi, and Plants) (Melbourne Code, 以下简称《墨尔本法 规》) (http://www.iapt-taxon.org/nomen/main.php) (McNeill et al, 2012)中, “后选模式”详见规则9.2和 词汇中的第157页, “原白”则在辅则8A.4的脚注和词 汇中的159页中有详细解释。

在规则9.2中是这样表述后选模式的: “A lectotype is a specimen or illustration designated from the original material as the nomenclatural type, in conformity with Art. 9.11 and 9.12, if no holotype was indicated at the time of publication, or if the holotype is missing, or if a type is found to belong to more than one taxon (see also Art. 9.14). For sanctioned names, a lectotype may be selected from among elements associated with either or both the protologue and the sanctioning treatment (Art. 9.10)”。可翻译为: “如果在发 表论文时(含发表新类群, 译者注), 原作者(译者注) 没有指明主模式、主模式丢失或发现该模式属于多 个分类群时(见规则9.14), 可从原始材料(见规则 9.3, 译者注)中指定标本或插图作为命名模式, 该标本 或插图即为后选模式。它与规则 9.11 和 9.12 保持一 致。就认可名称(sanctioned name)而言, 后选模式可 从其原白或/和认可处理的成分中选取”。

在辅则 8A.4 脚注 1 中是这样表述原白的: "Protologue (from Greek $\pi \rho \omega ́ \tau o \zeta$, protos, first; $\lambda$ ó $\gamma \circ \varsigma$, logos, discourse): everything associated with a name at its valid publication, e.g. description, diagnosis, illustrations, references, synonymy, geographical data, citation of specimens, discussion, and comments”。可 翻译为: “原白(源于希腊语, $\pi \rho \omega ́ \tau \sigma, 5$, protos为首次; $\lambda$ ó $05, \log 0 \mathrm{~s}$ 为短文): 与合格发表名称关联的任何 信息如描述、特征集要、插图、参考文献、异名、 地理资料、引证标本、讨论及评注”。

导致错误或重复指定后选模式的主要原因在 于作者未能全面掌握研究相关类群的文献。如果这 些错误不予纠正, 将会致使后来学者盲目遵从, 以 
讹传讹。根据规则 9.19, 首次基于规则9.11-9.13指 定的后选模式必须遵从，除非(a)主模式或选择新模 式后而其原始材料被重新找到, (b)后选模式与原白 严重不符或存在与原白一致的其他成分或(c)违反 规则 9.14 。现就以下案例逐一分析, 以便引起分类 学家足够的重视。

案例1 关于Pourthiaea Decne.的后选模式。 Liu和Hong (2016: 208) 提到: “Pourthiaea Decne. (1874: 146).-Type, designated here: Pourthiaea villosa (Thunb.) Decne. (1874: 147). Basionym: Crataegus villosa Thunb. (1784: 465)”。从字面上可以理 解为, Liu和Hong (1.c.)在这里为属Pourthiaea Decne. 指定了模式, 但具体是什么类型的模式, 读者不得 而知。根据规则7.10注解2, “designated here”仅适用 于2001年1月1日(含)的后选模式(lectotype)、新模式 (neotype)和附加模式(epitype), 由此判定Liu和Hong (1.c.) 为该属指定了后选模式。然而, Greuter等(1993: 917) 已经明确写道: “Pourthiaea Decne. in Nouv. Arch. Mus. Hist. Nat. 10: 125, 146. 1874.-Type: P. villosa (Thunb.) Decne. (Crataegus villosa Thunb.) [DICOTYLEDONES: ROSACEAE]”。根据规则9.2, Greuter等(1.c.)的模式指定应为后选模式指定(根据 规则10.1注解1)(在Pourthiaea Decne. 的原白中包含 有11种, Decaisne (1874: 125, 146-149)并未给该属 指定模式), 根据规则9.19, Greuter等(1.c.)的指定必 须遵从, Liu和Hong (1.c.)的后选模式指定显然是多 余的, 应予废弃。

案例2 关于水杉(Metasequoia glyptostroboides Hu \& W. C. Cheng)的后选模式。Metasequoia Hu \& W. C. Cheng作为保留名在规则 11.8 Ex. 32中已有详 细解释。根据规则 55.1 , 只要Metasequoia 是合格发 表的, 即使是非法名称, 其属下种只要满足合格发 表的条件, 也是合法的。Hu和Cheng (1948: 154)基 于现代植物发表新种水杉, 原白依次包括英文描述 (pp. 154-157)、引证标本(p. 157, 采集人及采集号分 别为C. J. Hsueh no 5, C. J. Hsueh no 5I, C. T. Hua no 2, C. T. Hua nos 179, 180, 181, C. T. Hua no 26 , S. Coll. s.n. 和S. Coll. 183)、插图(Plate 1)、中文描述 (pp. 160-161)和分布(Plate 2), 并注明论文发表日期 为 1948 年 5 月 15 日。尽管当时作者没有指定主模式, 但根据规则 40.1 和 40.2 , 该种仍然属于合格发表。 $\mathrm{Ma}$ 等(2005: 476)依据规则9.2, 从原始材料所引证
的标本中选择C. J. Hsueh no 5 的部分采集作为该种 的后选模式(存于NF), 该论文合格发表于 2005 年 6 月 17 日。由于忽略了 $\mathrm{Ma}$ 等(1.c.)的后选模式指定, Farjon (2005: 114)从原始材料中选择C. T. Hua no 2 标本, 重新为该种指定了后选模式(存于NF), 该论 著发表日期为2005年7月 8 日, 晚于前者21天。林祁 和曹子余(2007: 291)在没有看到Ma等(1.c.)和Farjon (1.c.)后选模式指定的情况下, 又从原始材料中选择 标本, 重新指定 C. J. Hsueh no 5 标本为其后选模式 (存于PE), 发表日期为 2007 年 2 月 10 日，明显晚于 前二者。只要 $\mathrm{Ma}$ 等(1.c.)的选择符合规则 9.19 , 他们 的选择必须遵从。Farjon (1.c.)与林祁和曹子余(1.c.) 的指定显然是无效的, 应予废弃。如果后人在研究 过程中忽略了 Ma等(1.c.)的指定, 而误引证Farjon (1.c.)或林祁和曹子余(1.c.) 的指定, 将会产生错误的 引证。

案例3 Hong (2015: 273) 写道: “13. Lobelia erectiuscula H. Hara, J. Jap. Bot. 40: 328.1965. $\equiv$ Lobelia erecta Hook. f. \& Thomson, Proc. J. Linn. Soc. Bot. (应为J. Proc. Linn. Soc. Bot.) 2: 28. 1857 (应为1858 '1857'), non De Vriese (1845)”。作者在此 为 Lobelia erectiuscula $\mathrm{H}$. Hara 指定了新模式 (neotype)。实际情况是: Lobelia erecta Hook. f. \& Thomson为晚出同名(Art. 53.1), 属于非法名称, 而 Lobelia erectiuscula H. Hara为此晚出同名的替代名 (Art. 6.11), 二者的模式是相同的(Art. 7.4)。只有在 Lobelia erecta Hook. f. \& Thomson的原白中的原始 材料丢失或不存在的情况下, 才有必要指定新模式 (Art. 9.13)。经考证, Lobelia erecta Hook. f. \& Thomson的原始材料(Art. 9.3)是“Hab. Himalayae orientalis regione temperatâ, Sikkim, alt. 9000-13000 ped.! (fl. August!) (v. v.), 且保存在K和GH。根据Art. 9.19, 此处的新模式指定是多余的、无效的, 应予废除。

致谢: 感谢中国科学院植物研究所杨永副研究员提 供部分水杉文献。

\section{参考文献}

Decaisne MJ (1874) Mémoire sur la famille des Pomacées. Nouvelles Archives du Muséum d'Histoire Naturelle, 10, 125, 146-149.

Farjon A (2005) A Monograph of Cupressaceae and Sciadopitys, 
p. 114. Royal Botanic Gardens, Kew.

Greuter W, Brummitt RK, Farr E, Kilian N, Kirk PM, Silva PC (1993) Names in Current Use for Extant Plant Genera, p.917. Koeltz Scientific Book, D-61453 Königstein, Germany.

Hong DY (2015) Lobelia L. In: Flora of Pan-Himalaya, Vol. 47 (ed. Hong DY), pp. 254-281. Science Press, Beijing.

$\mathrm{Hu} \mathrm{HH}$, Cheng WC (1948) On the new family Metasequoiaceae and on Metasequoia glyptostroboides, a living species of the genus Metasequoia found in Szechuan and Hupeh. Bulletin of the Fan Memorial Institute of Biology, 1, 153-162.

Lin Q, Cao ZY (2007) Lectotypifications of four names of Chinese taxa in Gymnospermae. Acta Botanica Yunnanica, 29, 291-292. (in Chinese with English abstract) [林祁, 曹 子余 (2007) 中国裸子植物四个植物名称的后选模式指 定. 云南植物研究, 29, 291-292.

Liu BB, Hong DY (2016) A taxonomic revision of the Pourthiaea villosa complex (Rosaceae). Phytotaxa, 244,
201-247.

Ma JS, Barringer K, Clemants S (2005) A revision of the typification of Metasequoia glyptostroboides (Taxodiaceae). Taxon, 54, 475-476.

McNeill J, Barrie FR, Buck WR, Demoulin V, Greuter W, Hawksworth DL, Herendeen PS, Knapp S, Marhold K, Prado J, Prud'homme van Reine WF, Smith GF, Wiersema JH, Turland NJ (2012) International Code of Nomenclature for Algae, Fungi, and Plants (Melbourne Code) Adopted by the Eighteenth International Botanical Congress, Melbourne, Australia, July 2011. Regnum Vegetabile Volume, 154, Koeltz Scientific Books, Germany.

Thunberg CP (1784) Systema Vegetabilium: Secundum Classes Ordines Genera Species cum Characteribus et Differentiis, 14 edn. (ed. Murray JA), p. 465. Gottingae, Germany.

(责任编委：严岳鸿 责任编辑：时意专) 